\title{
Temperature alters the shape of predator-prey cycles through effects on underlying mechanisms
}

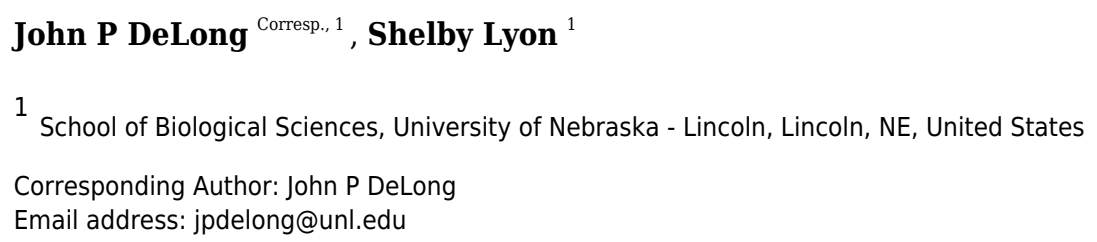

Background. Predicting the effects of climate warming on the dynamics of ecological systems requires understanding how temperature influences birth rates, death rates, and the strength of species interactions. The temperature dependence of these processes which are the underlying mechanisms of ecological dynamics - is often thought to be exponential or unimodal, generally supported by short-term experiments. However, ecological dynamics unfold over many generations. Our goal was to empirically document shifts in predator-prey cycles over the full range of temperatures that can possibly support a predator-prey system and then to uncover the effect of temperature on the underlying mechanisms driving those changes. Methods. We measured the population dynamics of the Didinium-Paramecium predator-prey system across a wide range of temperatures to reveal systematic changes in the dynamics of the system. We then used ordinary differential equation fitting to estimate parameters of a model describing the dynamics, and used these estimates to assess the long-term temperature dependence of all the underlying mechanisms. Results. We found that predator-prey cycles shrank in state space from colder to hotter temperatures and that both cycle period and amplitude varied with temperature. Model parameters showed mostly unimodal responses to temperature, with one parameter (predator mortality) increasing monotonically with temperature and one parameter (predator conversion efficiency) invariant with temperature. Our results indicate that temperature can have profound, systematic effects on ecological dynamics, and these can arise through diverse and simultaneous changes in multiple underlying mechanisms. Predicting the effects of temperature on ecological dynamics may require additional investigation into how the underlying drivers of population dynamics respond to temperature beyond a short-term, acute response. 
1 Temperature alters the shape of predator-prey cycles 2 through effects on underlying mechanisms

3

4

John P. DeLong ${ }^{1}$, Shelby Lyon ${ }^{1}$

1 School of Biological Sciences, University of Nebraska - Lincoln, Lincoln, NE, USA

Corresponding Author:

John DeLong ${ }^{1}$

${ }^{1}$ School of Biological Sciences, University of Nebraska - Lincoln, Lincoln, NE, USA Email address: jpdelong@unl.edu

\section{Abstract}

Background. Predicting the effects of climate warming on the dynamics of ecological systems requires understanding how temperature influences birth rates, death rates, and the strength of species interactions. The temperature dependence of these processes - which are the underlying mechanisms of ecological dynamics - is often thought to be exponential or unimodal, generally supported by short-term experiments. However, ecological dynamics unfold over many generations. Our goal was to empirically document shifts in predator-prey cycles over the full range of temperatures that can possibly support a predator-prey system and then to uncover the effect of temperature on the underlying mechanisms driving those changes.

Methods. We measured the population dynamics of the Didinium-Paramecium predator-prey system across a wide range of temperatures to reveal systematic changes in the dynamics of the system. We then used ordinary differential equation fitting to estimate parameters of a model describing the dynamics, and used these estimates to assess the long-term temperature dependence of all the underlying mechanisms.

Results. We found that predator-prey cycles shrank in state space from colder to hotter temperatures and that both cycle period and amplitude varied with temperature. Model parameters showed mostly unimodal responses to temperature, with one parameter (predator mortality) increasing monotonically with temperature and one parameter (predator conversion 
31 efficiency) invariant with temperature. Our results indicate that temperature can have profound,

32 systematic effects on ecological dynamics, and these can arise through diverse and

33 simultaneous changes in multiple underlying mechanisms. Predicting the effects of temperature

34 on ecological dynamics may require additional investigation into how the underlying drivers of

35 population dynamics respond to temperature beyond a short-term, acute response.

36

37 Introduction

Changes in environmental temperature have the potential to alter the dynamics and function of natural systems. With natural or human induced increases in temperature, biological rates including photosynthesis, respiration, movement, and reproduction typically increase, at least up to a point (Kleiber, 1961; Gillooly et al., 2001; Brown et al., 2004). These increases can have cascading effects throughout ecological communities by altering both individual phenotypes and the interactions that occur among individuals and species (O'Connor, 2009; Petchey, Brose \& Rall, - 2010; O’Connor, Gilbert \& Brown, 2011; Dell, Pawar \& Savage, 2014; Gilbert et al., 2014; Osmond et al., 2017). Although temperature clearly influences the biochemical processes operating within organisms (Johnson, 1962; Feller, 2010), how temperature simultaneously influences the full range of processes that govern the dynamics of complex ecological systems is far from understood.

Consumer-resource interactions are sensitive to temperature changes because both foraging rate per se and the strength of the consumer-resource interaction are driven by multiple temperature-dependent processes (Dell, Pawar \& Savage, 2014; Gilbert et al., 2014; Allan et al., 2015). This temperature dependence has strong implications for community dynamics and function because of the relationships among interaction strengths, population and community stability (e.g., the occurrence and shape of population cycles), and the overall structure of food webs (O'Connor et al., 2009; Petchey, Brose \& Rall, 2010; McCann, 2011; Binzer et al., 2012; Gilbert et al., 2014; Gibert et al., 2016; Gibert \& DeLong, 2017). Theory predicts a variety of changes in stability and dynamical behavior of consumer-resource communities with changes in 
57 temperature, depending upon assumptions about which processes are temperature-dependent

58 and the nature of that dependence (e.g., monotonically increasing or unimodal) (Vasseur \&

59 McCann, 2005; Ohlberger et al., 2011; O’Connor, Gilbert \& Brown, 2011; Binzer et al., 2012; Fussmann

60 et al., 2014; Amarasekare, 2015; Gibert et al., 2016; Osmond et al., 2017).

61 One often overlooked aspect of current theory predicting the effect of warming on

62 community dynamics is the mismatch between the temperature-dependence of rates and the

63 time-scale of the community dynamics. Most models assume that model parameters are a

64 function of temperature, using some function that describes a measured short-term effect of

65 temperature on biological rates. For example, the Boltzmann-Arrhenius function is often used to

66 invoke the kinetic effect of temperature on vital rates or interactions among species (Vasseur \&

67 McCann, 2005; Petchey, Brose \& Rall, 2010; O’Connor, Gilbert \& Brown, 2011; Fussmann et al., 2014;

68 Gilbert et al., 2014; Osmond et al., 2017; Wang et al., 2019). This temperature-dependence is then

69 assumed to persist in the same quantitative and qualitative way across many generations.

70 However, when organisms experience a new temperature, they may acclimate or show cross-

71 generational plasticity that alters their response to temperature (Alexander Jr \& McMahon, 2004;

72 Osmond et al., 2017; Luhring \& DeLong, 2017). Thus, models parameterized with short-term thermal

73 responses can produce mixed results when compared with an observed long-term response

74 (Yang et al., 2016). Thus, the emergence of community dynamics at different temperatures is

75 likely to reflect a population's response to temperature post-acclimation, when the temperature

76 dependence of biological processes may have changed.

77 In response to increasing temperature, populations may show changes in the amplitude or

78 period of cycles (Nelson, Bjørnstad \& Yamanaka, 2013; Meisner, Harmon \& Ives, 2014; Salt et al., 2017)

79 or the persistence or stability of the system (Beisner, McCauley \& Wrona, 1997; Jiang \& Kulczycki,

80 2004; Salt et al., 2017). These patterns of change in response to temperature appear to be driven

81 by shifts in organism performance and species interactions with temperature, but it is not clear 
82 whether the assumptions of temperature effects on parameters used in the theoretical literature 83 align well with the dynamics observed in the empirical literature. This uncertainty is in part due 84 to potential differences between long- and short-term responses to temperature. To resolve this 85 problem, we could measure species interactions in situ or infer them from the dynamics 86 themselves by determining what parameter values and model structures can reproduce the 87 observed dynamics.

88 Here we examine the predator-prey cycles of the ciliate Didinium nasutum (hereafter 89 Didinium) foraging on the ciliate Paramecium bursaria (hereafter Paramecium) as they pass 90 through a population cycle. The Didinium-Paramecium predator prey system is a classic 91 laboratory tool for studying predator-prey dynamics (Gause, 1934; Luckinbill, 1973; Salt, 1974; 92 Jost \& Ellner, 2000; Minter et al., 2011; Montagnes et al., 2012; DeLong \& Vasseur, 2013; Li et 93 al., 2013; Li \& Montagnes, 2015; Salt et al., 2017). The advantages of this system are clear: 94 short generation times, small spatial footprint, specialization of Didinium on Paramecium making for a very strong predator-prey interaction, and ease of estimating population abundances. This system has been used to reveal numerous aspects of predator-prey interactions, and, as with many other ciliates, the Didinium-Paramecium system is a useful model for understanding thermal biology across a wide range of temperatures (Weisse et al., 2002; Yang et al., 2013). The breadth of temperatures that a predator can tolerate is constrained by the breadth of temperature their prey can tolerate, unless alternative prey are available. Therefore, we measured the population dynamics of this system at six temperatures that span almost the full range of positive growth of $P$. bursaria (Luhring \& DeLong, 2017) to determine how the predatorprey cycle changes with temperature. We then used ordinary differential equation (ODE) fitting to estimate the model parameters (i.e., the mechanisms of change in this system) and show how the net effect of temperature on dynamics is linked to changes in model parameters. Finally, we evaluated how each parameter influences the dynamics on their own. Together, 
107 these results indicate a complex suite of temperature effects on both predator and prey that lead

108 to striking variation in ecological dynamics.

109

\section{Materials \& Methods}

111 We acquired Didinium from Carolina Biological Supply (Burlington, North Carolina), and we

112 isolated Paramecium from a pond at the Spring Creek Prairie Audubon Center southwest of

113 Lincoln, Nebraska, USA (Novich et al., 2014). Stock cultures of both species were maintained in

114 the laboratory at $23^{\circ} \mathrm{C}$ in medium made from protozoan concentrate (Carolina Biological Supply)

115 mixed with filtered and autoclaved pond water acquired from the source pond for Paramecium

116 (1:9 ratio of concentrate to water).

117 We assembled $6 \mathrm{~mL}$ microcosms in $60 \mathrm{~mm}$ diameter plastic Petri dishes with lids, with

118 predator and no-predator treatments. We randomly assigned microcosms to predator dishes

119 (six replicates) and predator free dishes (four replicates). For predator dishes, we added $5.9 \mathrm{~mL}$

of $40 \mu \mathrm{m}$ filtered Paramecium stock culture (initial density of $\sim 30$ cells per $\mathrm{mL}$ ) to each dish. We rinsed didinia in sterile medium and then added two individuals to each predator dish in a 0.1 $\mathrm{mL}$ aliquot. We added this same volume of rinse medium to each predator-free dish to control for possible microbial contributions from the Didinium stock culture to the experimental dishes. Predator free dishes contained $5.9 \mathrm{~mL}$ culture medium plus six paramecia transferred in a 0.05 $\mathrm{mL}$ aliquot. We assigned replicate dishes randomly to one of six temperatures $(17,20,23,25$, 27 , and $31^{\circ} \mathrm{C}$ ) and kept them in Percival incubators on a $12 \mathrm{~h}: 12 \mathrm{~h}$ light:dark schedule. day 18 . We took a $0.1 \mathrm{~mL}$ sample from each dish daily and replaced this with $0.1 \mathrm{~mL}$ sterile medium plus $0.1 \mathrm{~mL}$ autoclaved pond water to account for evaporation. We conducted a complete visual census of the Didinium population by scanning the entire dish through the

131 microscope. For Paramecium, we used a scaled sampling regime, counting the paramecia in

132 the $0.1 \mathrm{~mL}$ sample when abundant and conducting a complete census of paramecia in the 
133 dishes when they were rarer ( $<100$ cells) (DeLong \& Vasseur, 2012). We averaged densities

134 across replicates to create an average trajectory for each temperature. This provides a useful

135 smoothing effect that is often essential for differential equation fitting (Jost \& Ellner, 2000) and that

136 provides a data set with reduced sampling error and stochasticity induced noise. In a few

137 dishes, the Didinium population went extinct within a day or two, reducing our replicate

138 population numbers to $4,6,4,5,6$, and 3 at the temperatures of $17,20,23,25,27$, and $31^{\circ} \mathrm{C}$,

139 respectively. In one replicate at $23^{\circ} \mathrm{C}$, the Didinium population increase showed a pronounced

140 lag, while the Paramecium population showed a growth, crash, and regrowth, generating

141 deviations in dynamics well beyond the other replicates. We therefore excluded this replicate

142 from the analysis.

143 At the peak of each Didinium population trajectory, we photographed 7-19 individual didinia

144 with a Leica M165C microscope and digital camera, measured cell length and width, and

145 calculated cell volume using the formula for a prolate spheroid.

146 We used the following ODE model to describe the time series of Didinium population density

147 ( $C$, for consumer) and Paramecium population density ( $R$, for resource):

$148 \quad \frac{d R}{d t}=\left(r-r_{\text {slope }} R\right) R-\frac{a R C}{1+a h R+m(C-0.167)} \quad$ Equation 1a

$149 \frac{d C}{d t}=e \frac{a R C}{1+a h R+m(C-0.167)}-C\left(d e^{-R C} d\right) \quad$ Equation 1b,

150 In this model, $r$ is the maximum population growth rate of Paramecium, and $r_{\text {slope }}$ is the slope of

151 the relationship between population density and realized per capita growth rate. This expression

152 is equivalent to the logistic growth model, where $r_{\text {slope }}=r / K$, with $K$ being carrying capacity, and

153 is the typical form of population growth for Paramecium (Jiang \& Kulczycki, 2004; Gibert et al.,

154 2017). We chose this expression because fitting routines converge more easily with it than with

155 the logistic growth model. The two equations are linked by a type II functional response

156 (Beddington, 1975; DeAngelis, Goldstein \& O’Neill, 1975; Skalski \& Gilliam, 2001), where a is the space 
157 clearance rate of the Didinium (i.e., the volume of habitat cleared of prey per predator per time),

$158 h$ is the handling time for Didinium, $m$ is interference competition among Didinium, $e$ is the

159 efficiency of converting Paramecium into new Didinium, $d$ is the Didinium maximum death rate,

160 and $C_{d}$ sets the density-dependence of death rate. The C-0.167 term allows interference to go

161 to zero when there is only one predator (the density is 0.167 individuals per $\mathrm{mL}$ when there is

162 one predator in the dish). Several previous works suggest the necessity of including prey-

163 dependent mortality for Didinium dynamics (Minter et al., 2011; DeLong, Hanley \& Vasseur, 2014; Li

$164 \&$ Montagnes, 2015), and the function introduced here allows mortality rates to decline as prey

165 become more abundant.

166 Despite the fact that we cannot know with certainty what the right model is for the Didinium-

167 Paramecium interaction, our chosen model contains key components of all consumer-resource

168 interactions and displayed a high degree of compatibility with the data (see Results), suggesting

169 the model reflects real aspects of the thermal biology of this system. In general, ODE models

170 are particularly useful for protist microcosm dynamics, since cell division and death can happen

171 at any time and reproduction does not occur during discrete breeding periods. However, ODE

172 models have the downside of often predicting exceedingly low population abundances (much

173 less than one individual) from which populations can still rebound. These low abundances are

174 generally taken to reflect densities for populations with large spatial scales. In the case of whole

175 populations contained within microcosms, these low abundances can be thought of as

176 functionally zero.

177 We fit Equation 1 to the time series data using the Potterswheel toolbox version 4.1.6 in

178 Matlab 2017a (Raue et al., 2009; DeLong, Hanley \& Vasseur, 2014). We used the average time

179 series rather than individual replicate populations to avoid fitting stochasticity and noise in the

180 data sets and to aid in the identification of confidence intervals on the parameters. Fits to

181 individual replicate time series were possible for some replicates but not others, as stochasticity 
182 and limited number of observations made identifying a good model and robust parameter 183 estimates impossible in some cases. Furthermore, differences among replicates arose through 184 stochasticity, such that the variation across replicates reflects variation not caused by the 185 underlying deterministic drivers of the system, and it is these underlying mechanisms that are of 186 interest in this study. The Potterswheel fitting tool searches parameter space to identify 187 parameter sets for which the solution to Equation 1 provides a fit to the data for which further 188 changes in parameters do not lead to an improved fit. The fitting approach minimizes a $\chi^{2}$ 189 deviance across all measurements.

We used profile likelihood estimation to characterize uncertainty of the parameter estimates 191 (Raue et al., 2009). Profile likelihood calculates the parameter value for which an increase in the 192 model's $\chi^{2}$ goodness of fit statistic reaches a particular threshold. We set this threshold to a $19368.5 \%$ confidence interval $(\mathrm{Cl})$ because pushing the parameters farther than this from the mean 194 estimate frequently caused integration failure of the solvers. Thus, our uncertainty estimates are 195 approximately the standard deviation of the parameter (Raue et al., 2009). The profile-likelihood is 196 estimated in log increments, preventing negative confidence interval estimates. We prioritized 197 profile likelihood confidence intervals, but in cases where these were unattainable, we used Cls 198 estimated with the Hessian matrix of Equations 1 provided by the Potterswheel toolbox. 199 We first fit Equation 1 to each time series with all parameters unconstrained. This process 200 indicated that the conversion efficiency (parameter $e$ ) was very similar across temperatures 201 (mean $=0.055$, with Cls 0.028 to 0.097 inclusive of all temperatures). We tested whether fixing 202 the conversion efficiency to the mean value impaired fits, and at all temperatures, $\chi^{2}$ values 203 increased by only 0.2 to 6.4 , indicating little impact on fit quality. We therefore inferred that 204 conversion efficiency is somewhat invariant with temperature. We also determined that the 205 density-dependent mortality parameter $\left(C_{d}\right)$, while necessary to include given poor fits without it, 
206 was nonetheless very difficult to estimate. We detected good fits in the area of $C_{d}=40$, and we 207 also determined that $C_{d}$ could be fixed at 40 without loss of fit quality.

208 Previous evaluations demonstrated that the Potterswheel ODE fitting approach provides 209 robust parameter estimation without generation of spurious covaration among parameter 210 estimates (DeLong et al., 2018). Nonetheless, we evaluated the ability of our ODE fitting 211 methods to recover model parameters from dynamics. We did this in two steps. First, we used 212 ODE solvers to generate simulated model dynamics from Equation 1, and then second, we 213 used the same fitting routines as used in the main analysis to identify model parameters of 214 these simulated datasets. We repeated the testing for each of the six estimated parameter sets 215 (one set for each temperature). We also restricted the timespan of the test simulation to the time 216 frame of the observed dynamics, which includes all time steps with positive abundances and the 217 three time periods of zero abundance after the last non-zero abundance time point. At all six 218 temperatures, the ODE fitting returned exactly the parameters used to generate the simulations 219 (Figure S1). We repeated this analysis after introducing noise to the time series by adding a 220 number drawn from a random normal distribution $(\bar{x}=0 ; \sigma=0.1)$ and repeating the fitting process at each temperature 10 times. We were still able to recover parameters, albeit less exactly than without noise (Figure S2). All Matlab files required to conduct fitting and test parameter recovery are available in the supplementary materials.

Paramecium populations in the predator free control dishes increased in density through time, but these populations did not achieve an identifiable carrying capacity at all temperatures. We therefore used this data only to calculate rate of growth $r$ for Paramecium in predator free conditions. We extracted data from days 2 and 4 and used the standard exponential growth

228 model: $r=\frac{\ln \frac{N_{4}}{N_{2}}}{2}$, where $N_{2}$ and $N_{4}$ are population densities at time 2 and 4 , respectively. There 229 was a decline in density from the initial inoculation to day 2, so we did not use this first time 230 step. We calculated $r$ for each replicate separately to estimate error. 
Finally, we evaluated the effects of variation in parameters due to temperature on the dynamics. We first solved our model for the mean parameter set. We then varied each parameter on its own from the minimum to the maximum fitted values across temperatures and solved the model again using the mean fitted values for the other parameters. Thus, for each parameter we show three sets of dynamics reflecting three parameters sets: 1) the minimum parameter set contains the minimum fitted value of the focal parameter and the means for the other parameters, 2) the mean parameter set uses the mean of all parameters and thus is the same in all contrasts, and 3) the maximum parameter set contains the maximum fitted value of the focal parameter along with the means of all other parameters. These contrasts show how variation in specific parameters alters the predator-prey dynamics while holding the other parameters constant.

\section{Results}

243 The dynamics of the interacting Didinium-Paramecium populations showed a clear shift in

244 shape from colder to warmer temperatures (Figs 1-2). As temperature increased, the period of 245 the population cycle decreased, while the amplitude of the Didinium cycle increased and then decreased and the amplitude of the Paramecium cycle started to decline around $23^{\circ} \mathrm{C}$ (Figs $1 \mathrm{~A}$ B). In state space, these shifts were seen in a reduction in the radius of the trajectory as populations increased, decreased, and finally went extinct (Fig. 1C). The time to extinction decreased as temperature increased (Fig 1).

Based on our fitting results, we infer that six of the parameters governing these interactions changed with temperature (Fig. 3). Most parameters (Paramecium growth rate, Paramecium strength of density dependence, space clearance rate, interference, and handling time) showed a unimodal response, peaking at intermediate temperatures, typically near $27^{\circ} \mathrm{C}$, but at $20^{\circ} \mathrm{C}$ in

254 the case of handling time. In contrast, maximum Didinium mortality showed a monotonic 255 increase with temperature. Finally, in the predator free dishes, Paramecium rate of population 256 growth $r$ peaked at $23^{\circ} \mathrm{C}$, but overall, rate of growth for Paramecium was much lower in the 
257 predator-free dishes than in the presence of predators, especially at higher temperatures (Fig. $2583 A)$.

259 The shifts in dynamics in this system due to changes in temperature were influenced 260 strongly by all temperature-dependent parameters (Fig. 4). The changing amplitude in the 261 system (Fig. 1) was influenced by variation in Paramecium rate of growth and density 262 dependence as well as Didinium mortality. The shifts in period were more related to a 263 combination of the effect of temperature on space clearance rate, mutual interference, and 264 handling time (Figs 4G-H).

265 Didinium cell volume shifted with temperature. From the initial size $\left(4.8 \times 10^{-4} \mu \mathrm{m}^{3}\right.$ in stock 266 cultures maintained at $23^{\circ} \mathrm{C}$ ), mean Didinium cell volume increased with temperature from $17^{\circ} \mathrm{C}$ 267 to $21^{\circ} \mathrm{C}$ and then decreased with further increases in temperature (Fig. 5).

268

269 Discussion

270 Predicting how changes in temperature alter population and community dynamics depends on 271 developing a thorough understanding of how temperature alters the underlying drivers of 272 population growth and species interactions. Currently, however, numerous assumptions about 273 the temperature dependence of parameters governing species interactions are still required to 274 make predictions about the effects of warming on population and community dynamics. A more 275 complete depiction of the effect of temperature on underlying mechanisms driving population 276 dynamics is needed, especially over broad temperature ranges and taking into account 277 organismal acclimation. Here we used a combined theoretical-empirical approach to 278 characterize dynamics across temperature and uncover the temperature dependence of the 279 drivers of these patterns.

280 We found that the dynamics of the Didinium-Paramecium system shifted steadily as 281 temperature increased, from longer to shorter cycle periods and a change in amplitude (Figs 
283 moths (Adoxophyes honmai) as a result of increasing temperature (Nelson, Bjørnstad \&

284 Yamanaka, 2013), to the decreasing period and increasing amplitude of pea aphid (Acyrthosiphon

285 pisum)-parasitoid wasp (Aphidius ervi) cycles with increasing temperature (Meisner, Harmon \&

286 Ives, 2014), and to shifts in both period and amplitude of Didinium-Paramecium caudatum

287 dynamics (Salt et al., 2017). Yet by considering a broad range of temperatures, we also found

288 that some of these dynamic shifts themselves may be unimodal rather than monotonically

289 changing.

290 Our ODE fitting analysis revealed that the underlying drivers of these dynamics showed

291 pervasive responses to temperature (Figs 1-2). Most components of our model, including prey

292 births, the functional response, and predator mortality, showed some type of response to

293 temperature (Fig. 3). In contrast to expectations about the exponential (Arrhenius type) effect of

294 temperature on ecological processes (Brown et al., 2004; Dell, Pawar \& Savage, 2011; Burnside et al., 295 2014), only Didinium mortality rate showed an exponential-like increase from low temperature to 296 higher temperatures. Rather, most parameters showed a unimodal response, in line with other 297 observations about fecundity, population growth, and the functional response across 298 temperatures (Ratkowsky, Olley \& Ross, 2005; Englund et al., 2011; Amarasekare, 2015; Uszko et al., 299 2017; Uiterwaal \& DeLong, 2020). The typical peak or minimum temperature was $27^{\circ} \mathrm{C}$, such that 300 four of the five parameters shifted in a correlated manner as temperature changed. This pattern 301 also reflects the previously observed correlation between space clearance rate and interference 302 in the Didinium-Paramecium system (DeLong \& Vasseur, 2013). Thus, the actual dynamics of 303 these populations arose from a combination of monotonically increasing and unimodal 304 responses of ecological functions, which is consistent with some theoretical work (Amarasekare, 305 2015). By varying parameters one at a time, it appears that temperature effects on births and 306 death rates influenced changes in cycle amplitude, while temperature effects on the functional 307 response influenced changes in cycle period (Fig. 4). 
Our approach here was to infer the temperature dependence of the mechanisms driving population dynamics by fitting an ODE consumer-resource model (Equation 1) to data and comparing fitted parameters across temperature (DeLong et al., 2018). Although all parameter

311 estimates are model-dependent and thus to some degree sensitive to model selection, this

312 approach (the indirect approach, sensu Palamara et al. 2014) allowed us to uncover temperature dependencies that unfolded over the full period of the interaction. Although the shape of some of these relationships were quite clearly unimodal (e.g., maximum population growth rate), other cases were less clearly so (e.g., space clearance rate), suggesting the need for additional work to identify these relationships more precisely (Figure 3).

The predator-prey interaction in our experiment lasted about 3-25 Didinium generations (maximum generation time estimated as e/h) and 20-80 Paramecium generations (generation time estimated as $1 / r$ ). Over these generations, changes in Didinium or Paramecium physiology or morphology, through acclimation or phenotypic plasticity, could have had time to take effect and influence the outcome. For example, Didinium in our study showed a clear dependence of cell volume on temperature at the peak of their cycle (Fig. 5), partially consistent with the temperature-size rule (Atkinson, 1994; DeLong, 2012), although cell volume likely was also responding to the dynamic changes in resource levels through time (DeLong, Hanley \& Vasseur, 2014). Thus, our results reflect long-term rather than short-term responses to temperature.

In our study, both predator and prey populations went deterministically extinct by the end of the experiment at all temperatures, consistent with the typical behavior of Didinium and Paramecium without stabilizing environmental factors introduced to the microcosms (Luckinbill, 1973; DeLong \& Vasseur, 2013; Salt et al., 2017). Although extinctions occurred earlier in warmer temperatures, the system did not undergo major qualitative shifts such as from stable to unstable or from having a fixed point equilibrium to having oscillations, which are common 
333 predictions from theory (Vasseur \& McCann, 2005; Amarasekare, 2015). Rather, temperature

334 somewhat smoothly compressed the dynamics from a longer cycle through state space to one 335 that lasted only a few days (Fig. 1C).

336 We selected Equation 1 as a plausible model to use in our fitting routines. Although other 337 types of models could work, we emphasize that most of the terms and parameters in our model 338 are fundamental to predator-prey interactions and must be included in any type of model fitting in some manner. Parameterizing models such as Equation 1 with data taken from singlespecies laboratory observations is a useful way of dealing with the challenge of complex fitting problems such as the one here (DeLong, Hanley \& Vasseur, 2014). We conducted predator-free growth experiments for paramecia to identify some parameters outside of the ODE fitting task, but it was clear that in the presence of the predator, paramecia underwent cell divisions at a

344 much higher rate early in the experiment, especially at higher temperatures (Fig. 1A). Thus, we 345 could not fix Paramecium rate of growth with data from the single-species cultures to make 346 fitting easier and improve our estimates of other parameters. This difference is consistent with

347 recent observations that population growth thermal performance curves shift in the presence of 348 predators (Luhring \& DeLong, 2016), but it suggests that predicting the effects of temperature on predator-prey dynamics without in situ parameter estimates could prove misleading.

The difference between control and predator treatment Paramecium maximum rate of growth $(r)$ also was unexpected since the implied division rate of Paramecium at low densities was unusually high for a Paramecium-sized protist. We would anticipate that such a high $r$ would strictly occur at the lowest densities, with realized growth rates being much lower at even slightly higher population densities. It is also possible that the high growth rate was linked to short-term bursts of cell divisions arising from stored nutrients carried over from stock conditions or from reductions in cell volume. If this latter possibility occurred, smaller cells could alter the conversion efficiency of the predators, leading to compensating effects on the estimates of $r$. 
358 Untangling such shifts in parameters through time, and their impact on fitted parameter

359 estimates, may require greater effort to quantify traits such as cell size and processes such as

360 foraging rates in situ while tracking densities.

361

362

363

364

365

366

367

368

369

370

371

372

373

374

375

376

377

378

379

380

381

382

383

384

\section{Conclusions}

In conclusion, our results imply that there are few parameters that can be overlooked when seeking to predict shifts in population dynamics with changes in temperature. Although it is clear that shifts in the variance of temperature are likely to influence population outcomes (Estay, Lima \& Bozinovic, 2014; Vasseur et al., 2014), it is also clear that shifts in mean can have profound effects on community dynamics through direct effects on all of the mechanisms underlying such patterns. Our analysis focused on a specialized predator foraging on a single type of prey, but such tight species interactions might be quite rare in nature, with many predators having considerably broader diets than Didinium, including some protists (Roberts et al., 2010). It is therefore still unknown how the patterns of temperature dependence we observed here would manifest in a more diverse community with more generalist consumers. Nonetheless, as evidence amasses that changes in climate are altering the structure of the natural world and the dynamics of populations (Parmesan \& Yohe, 2003; Root et al., 2003; Tylianakis et al., 2008; Pol et al., 2010; Beck-Johnson et al., 2013), an assessment of the full range of temperature dependent mechanisms is needed.

\section{Acknowledgements}

Not applicable other than funders.

\section{References}

Alexander Jr JE, McMahon RF. 2004. Respiratory response to temperature and hypoxia in the zebra mussel Dreissena polymorpha. Comparative Biochemistry and Physiology Part A: Molecular \& Integrative Physiology 137:425-434. DOI: 10.1016/j.cbpb.2003.11.003. 
385 Allan BJM, Domenici P, Munday PL, McCormick MI. 2015. Feeling the heat: the effect of acute 386 temperature changes on predator-prey interactions in coral reef fish. Conservation Physiology 3. DOI: 10.1093/conphys/cov011.

Amarasekare P. 2015. Effects of temperature on consumer-resource interactions. Journal of Animal Ecology 84:665-679. DOI: 10.1111/1365-2656.12320.

Atkinson D. 1994. Temperature and organism size--a biological law for ectotherms? Advances in Ecological Research 25:1-58.

Beck-Johnson LM, Nelson WA, Paaijmans KP, Read AF, Thomas MB, Bjørnstad ON. 2013. The effect of temperature on Anopheles mosquito population dynamics and the potential for malaria transmission. PLOS ONE 8:e79276. DOI: 10.1371/journal.pone.0079276.

395

396

397

398

399

400

401

402

403

404

405

406

407

408

409

Beddington JR. 1975. Mutual interference between parasites or predators and its effect on searching efficiency. Journal of Animal Ecology 44:331-340.

Beisner BE, McCauley E, Wrona FJ. 1997. The influence of temperature and food chain length on plankton predator $\square$ prey dynamics. Canadian Journal of Fisheries and Aquatic Sciences 54:586-595. DOI: 10.1139/f96-312.

Binzer A, Guill C, Brose U, Rall BC. 2012. The dynamics of food chains under climate change and nutrient enrichment. Philosophical Transactions of the Royal Society B: Biological Sciences 367:2935-2944. DOI: 10.1098/rstb.2012.0230.

Brown J, Gillooly J, Allen A, Savage V, West G. 2004. Toward a metabolic theory of ecology. Ecology 85:1771-1789.

Burnside WR, Erhardt EB, Hammond ST, Brown JH. 2014. Rates of biotic interactions scale predictably with temperature despite variation. Oikos 123:1449-1456. DOI: 10.1111/oik.01199.

DeAngelis DL, Goldstein RA, O'Neill RV. 1975. A model for trophic interaction. Ecology 56:881892. 
410 Dell Al, Pawar S, Savage VM. 2011. Systematic variation in the temperature dependence of 411 physiological and ecological traits. Proceedings of the National Academy of Sciences 412 108:10,591-10,596. DOI: 10.1073/pnas.1015178108.

413 Dell AI, Pawar S, Savage VM. 2014. Temperature dependence of trophic interactions are driven 414 by asymmetry of species responses and foraging strategy. Journal of Animal Ecology 415 83:70-84. DOI: 10.1111/1365-2656.12081.

DeLong JP. 2012. Experimental demonstration of a 'rate-size' trade-off governing body size optimization. Evolutionary Ecology Research 14:343-352.

DeLong JP, Hanley TC, Gibert JP, Puth LM, Post DM. 2018. Life history traits and functional processes generate multiple pathways to ecological stability. Ecology 99:5-12. DOI:

421 DeLong JP, Hanley TC, Vasseur DA. 2014. Predator-prey dynamics and the plasticity of 422 predator body size. Functional Ecology 28:487-493. DOI: 10.1111/1365-2435.12199.

DeLong JP, Vasseur DA. 2012. Coexistence via resource partitioning fails to generate an increase in community function. PLoS ONE 7:e30081. DOI: 10.1371/journal.pone.0030081.

DeLong JP, Vasseur DA. 2013. Linked exploitation and interference competition drives the variable behavior of a classic predator-prey system. Oikos 122:1393-1400. DOI: 10.1111/j.1600-0706.2013.00418.x.

Englund G, Öhlund G, Hein CL, Diehl S. 2011. Temperature dependence of the functional response. Ecology Letters 14:914-921. DOI: 10.1111/j.1461-0248.2011.01661.x.

431 Estay SA, Lima M, Bozinovic F. 2014. The role of temperature variability on insect performance 432 433 and population dynamics in a warming world. Oikos 123:131-140. DOI: 10.1111/j.16000706.2013.00607.x. 
434 Feller G. 2010. Protein stability and enzyme activity at extreme biological temperatures. Journal

435

436

437

438

439

440

441

442

443

444

445

446

447

448

449

450

451

452

453

454

455

456

457

458

459

of Physics. Condensed Matter: An Institute of Physics Journal 22:323101. DOI: 10.1088/0953-8984/22/32/323101.

Fussmann KE, Schwarzmüller F, Brose U, Jousset A, Rall BC. 2014. Ecological stability in response to warming. Nature Climate Change 4:206-210. DOI: 10.1038/nclimate2134.

Gause GF. 1934. The Struggle for Existence. Williams and Wilkins, Baltimore (Reprinted 1964 by Hafner).

Gibert JP, Allen RL, Hruska RJ, DeLong JP. 2017. The ecological consequences of environmentally induced phenotypic changes. Ecology Letters 20:997-1003. DOI: 10.1111/ele.12797.

Gibert JP, Chelini M-C, Rosenthal MF, DeLong JP. 2016. Crossing regimes of temperature dependence in animal movement. Global Change Biology 22:1722-1736. DOI: 10.1111/gcb.13245.

Gibert JP, DeLong JP. 2017. Phenotypic variation explains food web structural patterns. Proceedings of the National Academy of Sciences 114:11187-11192. DOI: 10.1073/pnas.1703864114.

Gilbert B, Tunney TD, McCann KS, DeLong JP, Vasseur DA, Savage V, Shurin JB, Dell AI, Barton BT, Harley CDG, Kharouba HM, Kratina P, Blanchard JL, Clements C, Winder M, Greig HS, O'Connor MI. 2014. A bioenergetic framework for the temperature dependence of trophic interactions. Ecology Letters 17:902-914. DOI: 10.1111/ele.12307.

Gillooly JF, Brown JH, West GB, Savage VM, Charnov EL. 2001. Effects of size and temperature on metabolic rate. Science 293:2248-2251. DOI: 10.1126/science.1061967.

Jiang L, Kulczycki A. 2004. Competition, predation and species responses to environmental change. Oikos 106:217-224. DOI: 10.1111/j.0030-1299.2004.13056.x. 
460 Johnson B. 1962. Influence of temperature on the respiration and metabolic effectiveness of $461 \quad$ Chilomonas. Experimental Cell Research 28:419-423.

462 Jost C, Ellner SP. 2000. Testing for predator dependence in predator-prey dynamics: a non463 parametric approach. Proceedings: Biological Sciences 267:1611-1620.

464 Kleiber M. 1961. The Fire of life : an introduction to animal energetics / Max Kleiber. New York : $465 \quad$ Wiley.

466 Li J, Fenton A, Kettley L, Roberts P, Montagnes DJS. 2013. Reconsidering the importance of 467 the past in predator-prey models: both numerical and functional responses depend on 468 delayed prey densities. Proceedings of the Royal Society B: Biological Sciences 469 280:20131389. DOI: 10.1098/rspb.2013.1389.

470 Li J, Montagnes DJS. 2015. Restructuring fundamental predator-prey models by recognising 471 prey-dependent conversion efficiency and mortality rates. Protist 166:211-223. DOI:

472 10.1016/j.protis.2015.02.003.

473 Luckinbill LS. 1973. Coexistence in laboratory populations of Paramecium aurelia and its 474 predator Didinium nasutum. Ecology 54:1320-1327. DOI: 10.2307/1934194.

475 Luhring TM, DeLong JP. 2016. Predation changes the shape of thermal performance curves for 476 population growth rate. Current Zoology 62:501-505. DOI: 10.1093/cz/zow045.

Luhring TM, DeLong JP. 2017. Scaling from metabolism to population growth rate to understand 478 how acclimation temperature alters thermal performance. Integrative and Comparative Biology 57:103-111. DOI: 10.1093/icb/icx041.

McCann KS. 2011. Food Webs. Princeton University Press.

481 Meisner MH, Harmon JP, Ives AR. 2014. Temperature effects on long-term population 482 dynamics in a parasitoid-host system. Ecological Monographs 84:457-476. DOI: 10.1890/13-1933.1. 
484 Minter EJA, Fenton A, Cooper J, Montagnes DJS. 2011. Prey-dependent mortality rate: a critical

485

486

487

488

489

490

491

492

493

494

495

496

497

498

499

500

501

502

503

504

505

506

507 parameter in microbial models. Microbial Ecology 62:155-161. DOI: 10.1007/s00248011-9836-5.

Montagnes D, Roberts E, Lukeš J, Lowe C. 2012. The rise of model protozoa. Trends in Microbiology 20:184-191. DOI: 10.1016/j.tim.2012.01.007.

Nelson WA, Bjørnstad ON, Yamanaka T. 2013. Recurrent insect outbreaks caused by temperature-driven changes in system stability. Science 341:796-799. DOI: 10.1126/science.1238477.

Novich RA, Erickson EK, Kalinoski RM, DeLong JP. 2014. The temperature independence of interaction strength in a sit-and-wait predator. Ecosphere 5:art137. DOI: 10.1890/ES1400216.1.

O'Connor MI. 2009. Warming strengthens an herbivore-plant interaction. Ecology 90:388-398.

O'Connor MI, Gilbert B, Brown CJ. 2011. Theoretical predictions for how temperature affects the dynamics of interacting herbivores and plants. The American naturalist 178:626-638. DOI: 10.1086/662171.

O’Connor MI, Piehler MF, Leech DM, Anton A, Bruno JF. 2009. Warming and resource availability shift food web structure and metabolism. PLoS biology 7:e1000178. DOI: 10.1371/journal.pbio.1000178.

Ohlberger J, Edeline E, Vøllestad LA, Stenseth NC, Claessen D. 2011. Temperature-driven regime shifts in the dynamics of size-structured populations. The American Naturalist 177:211-223. DOI: 10.1086/657925.

Osmond MM, Barbour MA, Bernhardt JR, Pennell MW, Sunday JM, O’Connor MI, Kerkhoff AJ, Bronstein JL. 2017. Warming-induced changes to body size stabilize consumer-resource dynamics. The American Naturalist 189:718-725. DOI: 10.1086/691387.

Peer) reviewing PDF | (2020:01:44959:3:0:NEW 12 May 2020) 
508 Palamara GM, Childs DZ, Clements CF, Petchey OL, Plebani M, Smith MJ. 2014. Inferring the 509 temperature dependence of population parameters: the effects of experimental design 510 and inference algorithm. Ecology and Evolution 4:4736-4750. DOI: 10.1002/ece3.1309.

511 Parmesan C, Yohe G. 2003. A globally coherent fingerprint of climate change impacts across 512 natural systems. Nature 421:37-42. DOI: 10.1038/nature01286.

513 Petchey OL, Brose U, Rall BC. 2010. Predicting the effects of temperature on food web $514 \quad$ connectance. Philosophical Transactions of the Royal Society of London B: Biological $515 \quad$ Sciences 365:2081-2091. DOI: 10.1098/rstb.2010.0011.

516 Pol M van de, Vindenes Y, Sæther B-E, Engen S, Ens BJ, Oosterbeek K, Tinbergen JM. 2010.

$517 \quad$ Effects of climate change and variability on population dynamics in a long-lived 518 shorebird. Ecology 91:1192-1204. DOI: 10.1890/09-0410.1.

519 Ratkowsky DA, Olley J, Ross T. 2005. Unifying temperature effects on the growth rate of 520 bacteria and the stability of globular proteins. Journal of Theoretical Biology 233:351-

521 362. DOI: 10.1016/j.jtbi.2004.10.016.

Raue A, Kreutz C, Maiwald T, Bachmann J, Schilling M, Klingmüller U, Timmer J. 2009.

523 Structural and practical identifiability analysis of partially observed dynamical models by 524 exploiting the profile likelihood. Bioinformatics 25:1923-1929. DOI:

525 10.1093/bioinformatics/btp358.

Roberts EC, Wootton EC, Davidson K, Jeong HJ, Lowe CD, Montagnes DJS. 2010. Feeding in 527 the dinoflagellate Oxyrrhis marina: linking behaviour with mechanisms. Journal of Plankton Research 33:603-614. DOI: 10.1093/plankt/fbq118.

Root TL, Price JT, Hall KR, Schneider SH, Rosenzweig C, Pounds JA. 2003. Fingerprints of global warming on wild animals and plants. Nature 421:57-60. DOI: 10.1038/nature01333.

Salt GW. 1974. Predator and prey densities as controls of the rate of capture by the predator Didinium nasutum. Ecology 55:434-439. 
534 Salt JL, Bulit C, Zhang W, Qi H, Montagnes DJS. 2017. Spatial extinction or persistence:

535 landscape-temperature interactions perturb predator-prey dynamics. Ecography

$536 \quad$ 40:1177-1186. DOI: 10.1111/ecog.02378.

537 Skalski GT, Gilliam JF. 2001. Functional responses with predator interference: viable

$538 \quad$ alternatives to the Holling type II model. Ecology 82:3083-3092.

539 Tylianakis JM, Didham RK, Bascompte J, Wardle DA. 2008. Global change and species

$540 \quad$ interactions in terrestrial ecosystems. Ecology Letters 11:1351-1363. DOI:

$541 \quad$ 10.1111/j.1461-0248.2008.01250.x.

542 Uiterwaal SF, DeLong JP. 2020. Functional responses are maximized at intermediate

$543 \quad$ temperatures. Ecology 101:e02975. DOI: 10.1002/ecy.2975.

544 Uszko W, Diehl S, Englund G, Amarasekare P. 2017. Effects of warming on predator-prey

545 interactions - a resource-based approach and a theoretical synthesis. Ecology Letters

$546 \quad$ 20:513-523. DOI: 10.1111/ele.12755.

547 Vasseur DA, DeLong JP, Gilbert B, Greig HS, Harley CDG, McCann KS, Savage V, Tunney TD,

548 O'Connor MI. 2014. Increased temperature variation poses a greater risk to species than

549 climate warming. Proceedings of the Royal Society B: Biological Sciences

$550 \quad$ 281:20132612. DOI: $10.1098 /$ rspb.2013.2612.

551 Vasseur DA, McCann KS. 2005. A mechanistic approach for modeling temperature-dependent

552 consumer-resource dynamics. The American Naturalist 166:184-198. DOI:

$553 \quad 10.1086 / 431285$.

554 Wang Q, Lyu Z, Omar S, Cornell S, Yang Z, Montagnes DJS. 2019. Predicting temperature

555 impacts on aquatic productivity: Questioning the metabolic theory of ecology's

556 "canonical" activation energies. Limnology and Oceanography 64:1172-1185. DOI:

$557 \quad$ 10.1002/Ino.11105. 
558 Weisse T, Stadler P, Lindström ES, Kimmance SA, Montagnes DJS. 2002. Interactive effect of 559 temperature and food concentration on growth rate: a test case using the small 560 freshwater ciliate Urotricha farcta. Limnology and Oceanography 47:1447-1455.

561 Yang Z, Lowe CD, Crowther W, Fenton A, Watts PC, Montagnes DJS. 2013. Strain-specific 562 functional and numerical responses are required to evaluate impacts on predator-prey 563 dynamics. The ISME journal 7:405-416. DOI: 10.1038/ismej.2012.117.

564 Yang Z, Zhang L, Zhu X, Wang J, Montagnes DJS. 2016. An evidence-based framework for 565 predicting the impact of differing autotroph-heterotroph thermal sensitivities on 566 consumer-prey dynamics. The ISME Journal 10:1767-1778. DOI:

$567 \quad 10.1038 /$ ismej.2015.225.

568 
Figure 1

Dynamics of interacting Didinium nasutum and Paramecium bursaria populations.

Points are population densities averaged across replicates on each day. Panels on the left show how the dynamics change for each population with temperature separately, with (A) showing Paramecium and (B) showing Didinium. Panel (C) shows the state space trajectories for the populations together.
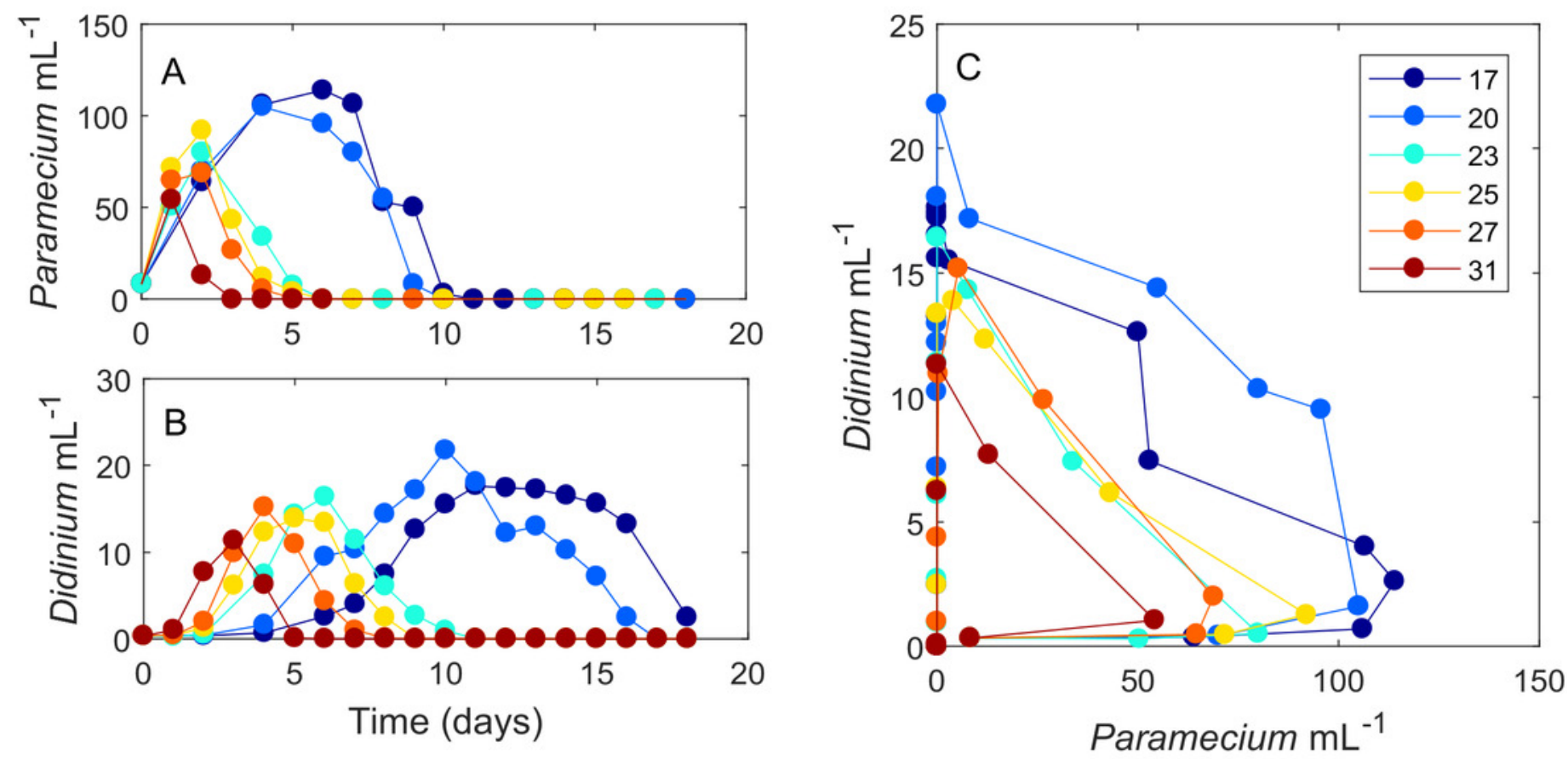
Figure 2

Dynamics of interacting Didinium nasutum and Paramecium bursaria populations.

Points are population densities averaged across replicates on each day. Top row (panels A-F) are Paramecium bursaria and bottom row (panels $\mathbf{G}-\mathbf{L}$ ) are Didinium nasutum. Temperatures are shown from cool to warm colors from left to right. Shaded areas represent SE of the across-replicate mean at each time point, and the heavy lines show fits of our model (Equation 1) to the data.
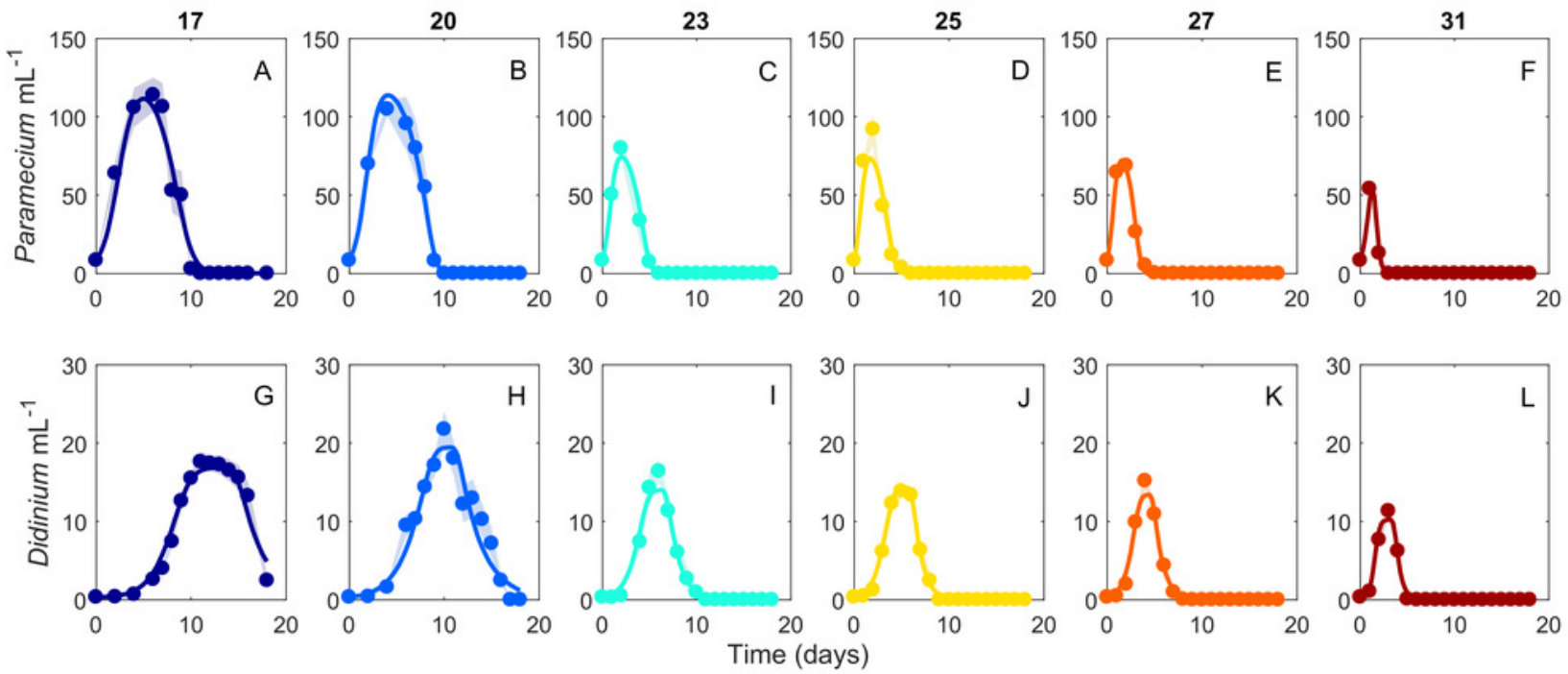


\section{Figure 3}

Estimated parameters from the fits of our ODE model (Equation 1) to the average dynamics of Didinium nasutum consuming Paramecium bursaria.

Solid dots indicate estimate and vertical lines indicate $68.5 \%$ confidence intervals (equivalent to one standard deviation). Parameters are $\mathbf{A}$ ) intrinsic rate of growth of Paramecium, B) strength of density dependence in Paramecium, C) space clearance rate, D) mutual interference, E) handling time, F) conversion efficiency, G) Didinium maximum mortality rate, and $\mathbf{H}$ ) half-saturation constant for Didinium prey-dependent mortality. $\ln \mathbf{A}$, open circles are the measured intrinsic rate of growth for Paramecium in the predator-free dishes. 

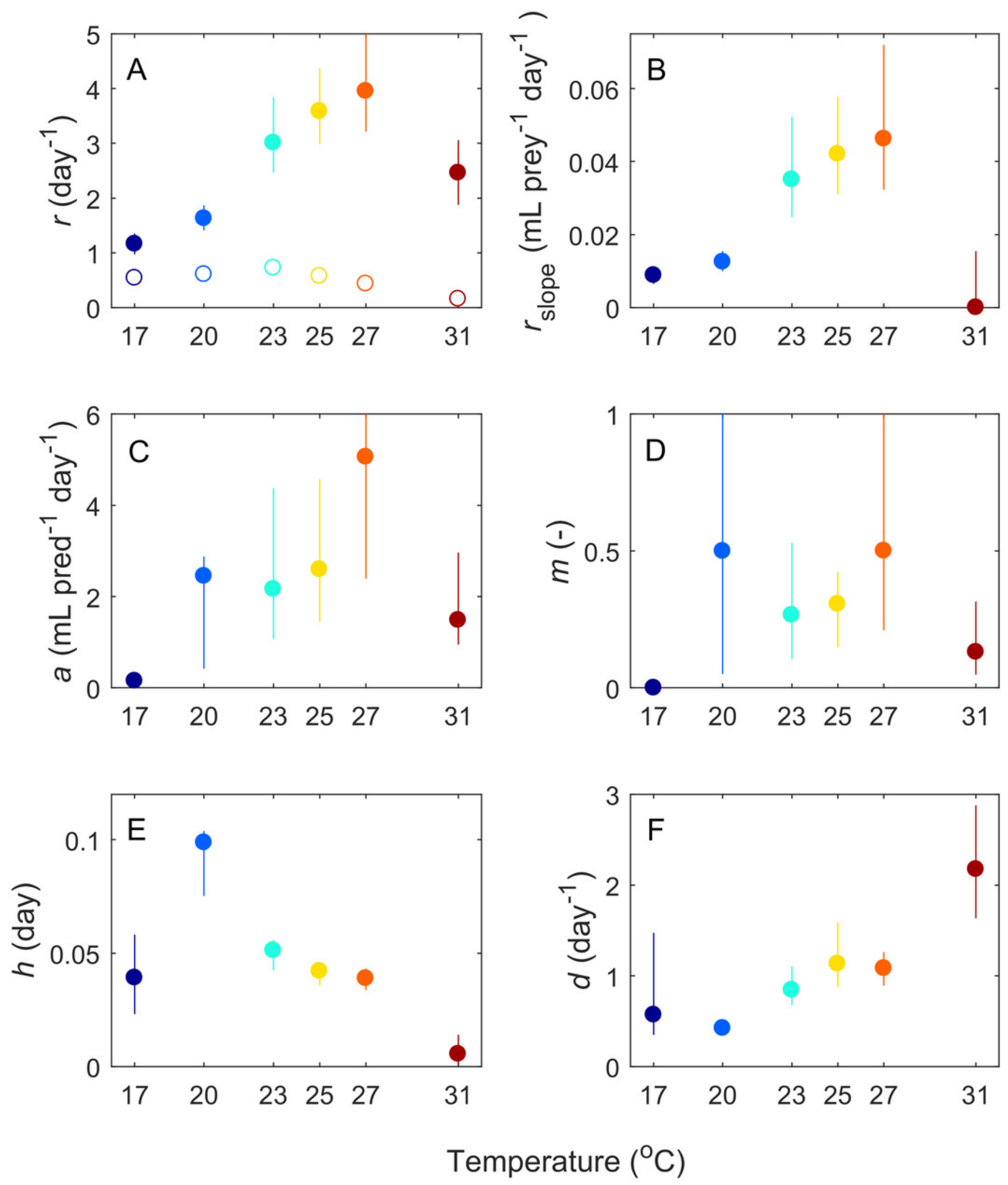


\section{Figure 4}

Changes in population dynamics due to variation in model parameters.

Each set of panels shows variation in population dynamics caused by variation in a single parameter, with other parameters held constant at the average of the fitted values across temperature. Panels show intrinsic rate of growth of Paramecium for prey (A) and predators (B), strength of density dependence in Paramecium for prey (C) and predators (D), space clearance rate for prey $(\mathbf{E})$ and predators $(\mathbf{F})$, mutual interference for prey $(\mathbf{G})$ and predators (H), handling time for prey (I) and predators (J), and Didinium maximum mortality rate for prey $(\mathbf{K})$ and predators ( $\mathbf{L})$. The lines show the trajectories for the Paramecium (solid line) and the Didinium (dashed line) populations for the mean parameter set (gray lines), the maximum parameter set (yellow lines), and the minimum parameter set (brick red lines). The maximum and minimum estimated parameters do not generally correspond to the extremes of temperature. 

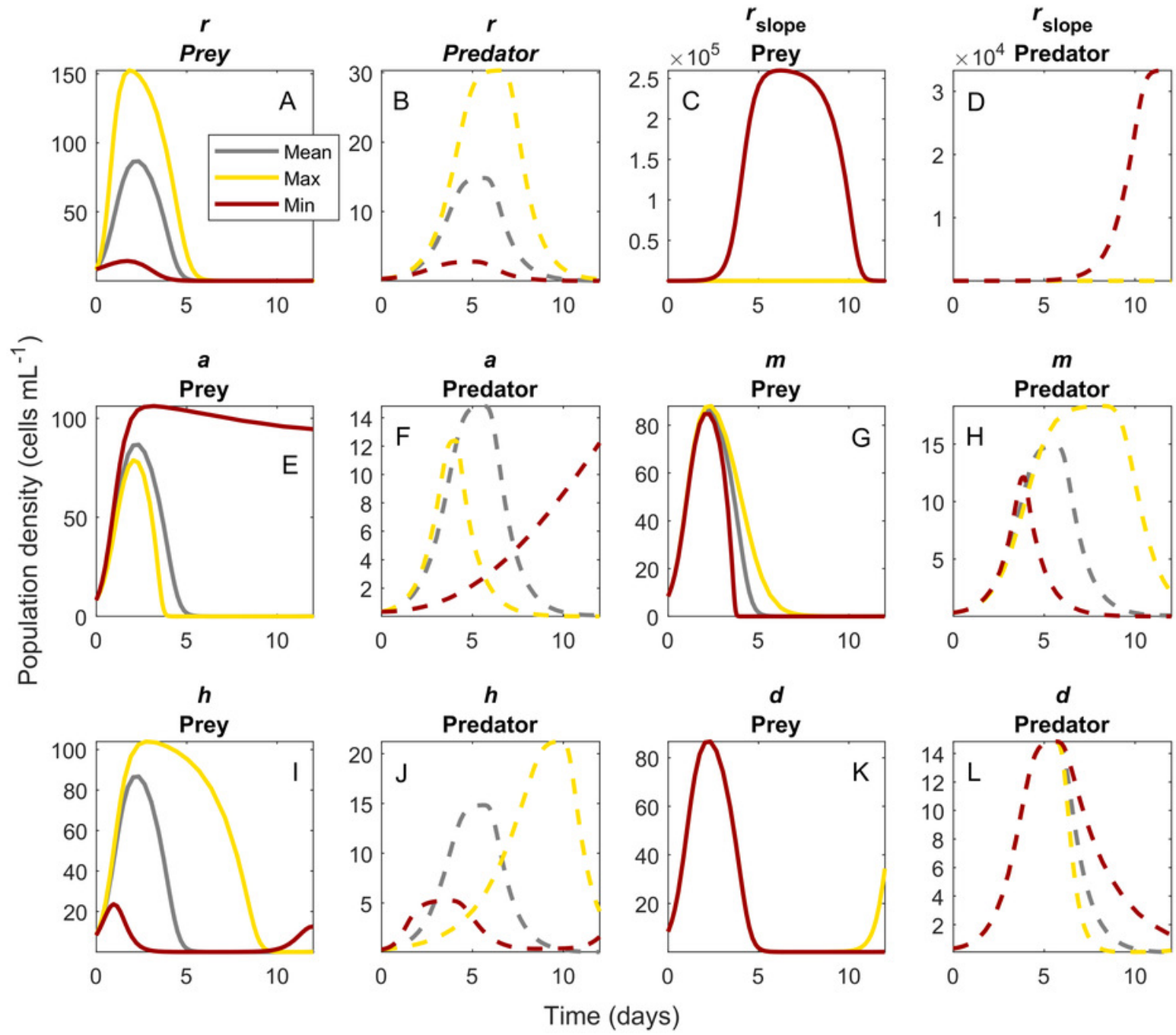
Figure 5

Mean Didinium nasutum cell volume at the top of their population cycle for each temperature.

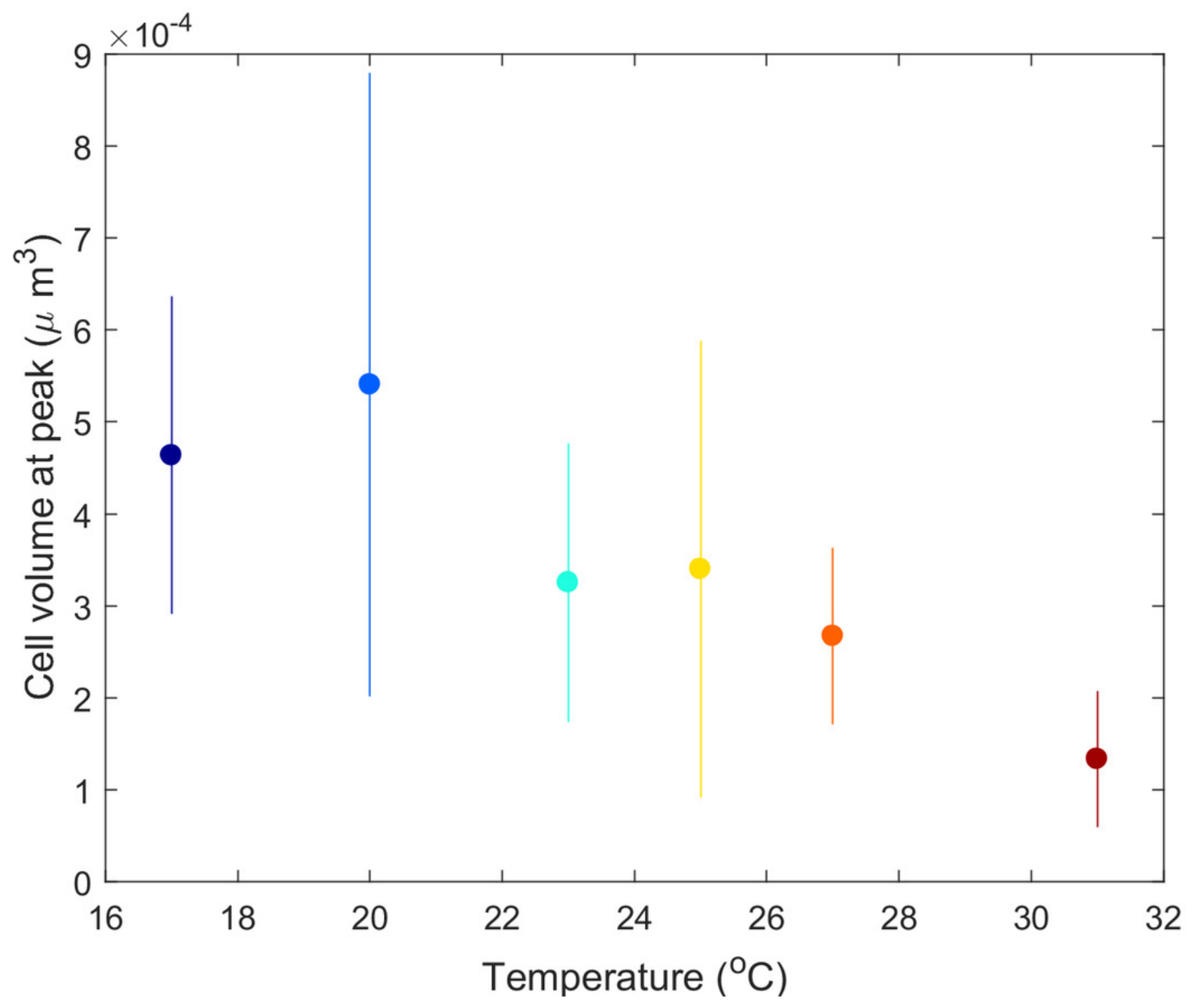

